\title{
Occult cancer diagnosed following acute ischemic stroke
}

\author{
Bastien Rioux MD, Mark R. Keezer MDCM PhD, Laura C. Gioia MD MSC
}

- Cite as: CMAJ 2020 September 8;192:E1037-9. doi: 10.1503/cmaj.200725

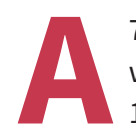

77-year-old right-handed functionally independent woman presented to the emergency department about 12 hours after the acute onset of speech difficulties. The patient was a nonsmoker and was receiving treatment for hypercholesterolemia (low-density lipoprotein cholesterol level at presentation $2.48 \mathrm{mmol} / \mathrm{L}$ [target $<2.0 \mathrm{mmol} / \mathrm{L}$ or $>50 \%$ reduction on treatment) with a moderate dose of atorvastatin, $20 \mathrm{mg} / \mathrm{d}$. She had no other modifiable cerebrovascular risk factors and no personal history of cancer or thromboembolic events. Although a sibling had been diagnosed with colorectal cancer at age 65 years, the patient had never undergone any screening for colorectal cancer. On examination, she had mild aphasia and right pronator drift. A noncontrast head computed tomography (CT) scan showed an ischemic nonlacunar infarct of the superior and middle left temporal gyri. We admitted her to our stroke unit for management and etiologic investigation.

The patient was afebrile, and there was no evidence of infection on chest radiography or urinalysis. Initial blood investigation showed mild normocytic anemia (hemoglobin concentration $116 \mathrm{~g} / \mathrm{L}$ [normal 120-160 g/L], mean corpuscular volume $90.2 \mathrm{fL}$ [normal 82.0-101.0 fL], normal serum ferritin level and a mildly elevated serum C-reactive protein level $(18.6 \mathrm{mg} / \mathrm{L}$ [normal $<10.0 \mathrm{mg} / \mathrm{L}]$ ). Her international normalized ratio and partial thromboplastin time were both within normal limits.

Twenty-four-hour ambulatory electrocardiography did not reveal atrial fibrillation, and transthoracic echocardiography showed mild left atrial enlargement, mild left ventricular diastolic dysfunction and nonsevere $(\leq 5 \mathrm{~mm})$ atheroma of the transverse aorta. Further investigations included brain magnetic resonance imaging, which corroborated the CT findings without additional ischemic lesions, and head and neck CT angiography, which did not show significant intracranial or extracranial stenosis.

Given the patient's mild anemia and elevated C-reactive protein level, we suspected occult cancer. We ordered chestabdomen-pelvis positron emission tomography/CT, which showed a large hypermetabolic mass involving the transverse colon (Figure 1). Subsequent contrast-enhanced abdomenpelvis CT failed to identify the lesion but, instead, revealed an additional, bulging mass involving the inner bladder wall (Figure 2). Colonoscopy confirmed the presence of an ulcerated infiltrative circumferential lesion located in the right portion of

\section{KEY POINTS}

- Acute ischemic stroke may be the first clinical manifestation of occult cancer.

- A comprehensive diagnostic approach to the causes of ischemic stroke may help identify uncommon etiologies, including occult cancer.

- Ensure age- and sex-appropriate cancer screening tests are up to date in survivors of acute ischemic stroke.

the transverse colon. The pathologic findings were consistent with low-grade colorectal adenocarcinoma (stage III, T3N1M0). Cystoscopy confirmed the presence of a bladder lesion, which was found to be stage I, low-grade urothelial bladder carcinoma.

Following surgical removal of her colorectal cancer, the patient received adjuvant chemotherapy with orally administered capecitabine, and her bladder cancer was resected cystoscopically. The bladder cancer recurred locally 13 months later, and the patient had a second bladder resection procedure. Twenty-two months later, she remained recurrence-free.

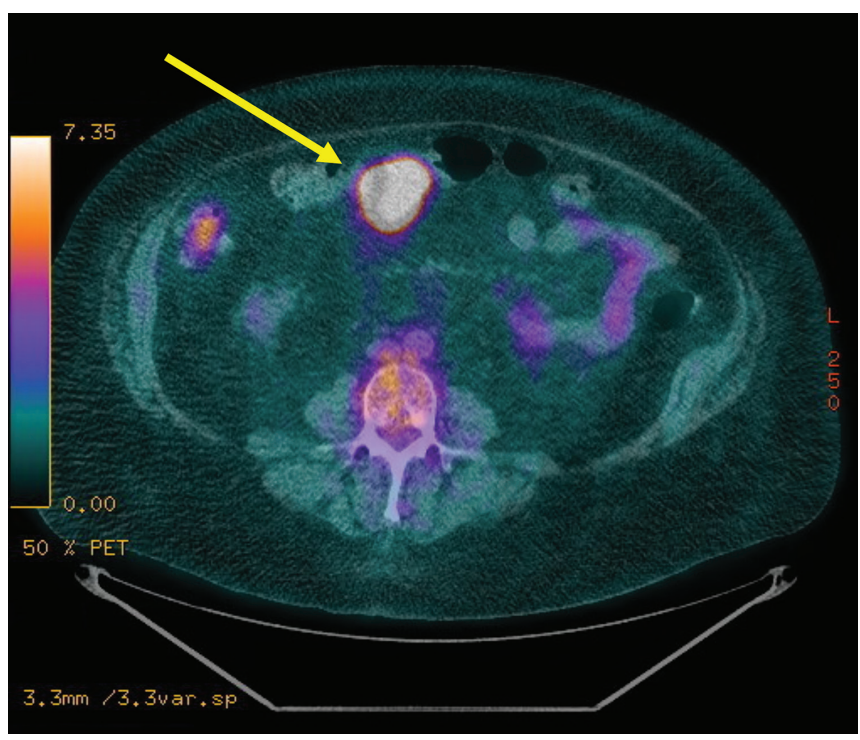

Figure 1: Axial positron emission tomography-computed tomography scan of the abdomen of a 77-year-old woman with acute ischemic stroke, showing a large hypermetabolic mass involving the transverse colon (arrow). 


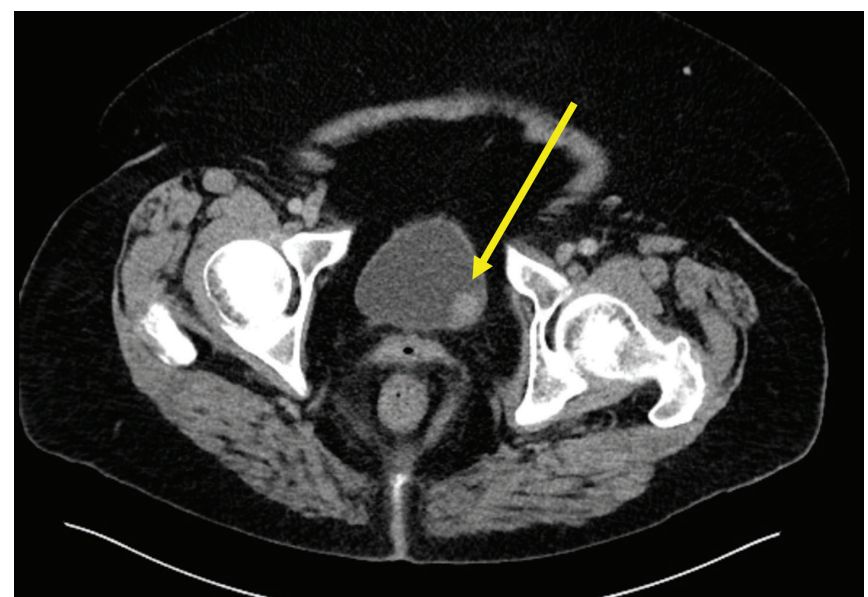

Figure 2: Contrast-enhanced axial abdomen-pelvis computed tomography scan, showing a bulging mass involving the inner bladder wall (arrow).

The patient attended an outpatient rehabilitation program for her stroke, where lifestyle modifications regarding diet and exercise were recommended. We prescribed low-dose acetylsalicylic acid $(80 \mathrm{mg} / \mathrm{d})$ and an angiotensin-converting enzyme inhibitor (perindopril, $4 \mathrm{mg} / \mathrm{d}$ ) for hypertension, and increased the dose of atorvastatin to $40 \mathrm{mg} / \mathrm{d}$. The patient remained fully independent after 3 years of follow-up, without any further ischemic events or other identified causes of stroke.

\section{Discussion}

We present the case of a 77-year-old woman with an acute ischemic stroke, possibly related to 2 occult malignant lesions diagnosed shortly after her index event. About $4 \%$ of people who experience acute ischemic stroke will receive a new cancer diagnosis in the subsequent 2 years, with older age, a history of smoking and an elevated D-dimer level conferring substantial risk. ${ }^{1}$ In a prospective cohort study, Qureshi and colleagues ${ }^{2}$ estimated a $20 \%$ increase in the age-adjusted cancer rate for the year following an ischemic stroke. Men are at higher risk than women for a poststroke cancer diagnosis. ${ }^{2}$

The pathophysiology underpinning the association between acute ischemic stroke and cancer is poorly understood. However, cancer-associated hypercoagulability and inflammation may promote acute ischemic stroke through several mechanisms, such as paroxysmal embolism, nonbacterial thrombotic endocarditis and intravascular coagulation. ${ }^{3}$ Furthermore, adenocarcinomas may secrete mucin into the bloodstream, which facilitates the formation of platelet-rich microthrombi by interacting with cell adhesion molecules. ${ }^{3}$

When acute ischemic strokes are associated with occult cancer, they tend to recur more often and confer a worse prognosis than strokes that are not associated with cancer. ${ }^{4}$ An acute ischemic stroke may be regarded as an opportunity to search more actively for cancer in selected cases and to improve the prognosis related to both stroke and malignant disease. ${ }^{3}$ However, the optimal diagnostic approach to occult cancer in patients with acute ischemic stroke is unclear. ${ }^{3}$ For example, current Canadian Stroke Best Practice and American Heart Association/American
Box 1: Cancer screening recommendations as per the Canadian Task Force on Preventive Health Care guidelines $^{7}$

\section{Colorectal cancer}

- For people aged 50-74 yr, fecal occult blood test every 2 yr or flexible sigmoidoscopy every $10 \mathrm{yr}$

\section{Lung cancer}

- For people aged 55-74 yr with $a \geq 30$ pack-year smoking history who currently smoke or who quit $<15$ years ago, low-dose computed tomography every year up to 3 consecutive times

- Routine screening with chest radiography is not recommended

\section{Breast cancer}

- For women aged 50-69 yr, mammography every 2-3 yr

- For women aged 70-74 yr, mammography every 2-3 yr; the decision to undergo screening is conditional on the relative value that a woman places on possible benefits and harms from screening

\section{Cervical cancer}

- For women aged 25-69 yr, Papanicolaou test every $3 \mathrm{yr}$

- For women aged $\geq 70 \mathrm{yr}$ who have not been adequately screened, continued screening until 3 negative Papanicolaou test results have been obtained

\section{Prostate cancer}

- Routine screening with prostate-specific antigen test is not recommended

*Screening should be carried out only in health care settings with expertise in early diagnosis and treatment of lung cancer.

Stroke Association recommendations on the secondary prevention of stroke do not provide guidance on when to suspect and investigate for occult malignant disease. ${ }^{5,6}$ Nonetheless, clinicians should think of occult cancer as part of a broader differential diagnosis for acute ischemic stroke.

In addition to reviewing the patient's history of age- and sexappropriate cancer screening tests (Box 1), clinicians may want to pay attention to risk factors (e.g., smoking, family history) and to symptoms (e.g., fever, night sweats, weight loss, rectorrhagia) and signs (e.g., cachexia, adenopathy) associated with cancer. An identified stroke cause does not exclude occult cancer. However, a higher proportion of patients with cryptogenic strokes than those with an identified cause of stroke have occult cancer, ${ }^{8}$ and most cancer-associated ischemic strokes are also associated with atrial fibrillation or large-vessel disease. ${ }^{9}$ Recommendations for cancer screening in the general population apply equally to survivors of acute ischemic stroke, and ensuring adequate screening may reduce cancer-related death in this group. ${ }^{7}$

Several markers of systemic inflammation (e.g., high C-reactive protein and fibrinogen levels, low albumin and hemoglobin levels) and upregulated coagulation activity (e.g., high D-dimer levels) are associated with occult cancer in acute ischemic stroke (Box 2). C-reactive protein levels greater than $20 \mathrm{mg} / \mathrm{L}$, when associated with acute ischemic stroke of undetermined cause, are predictive of occult cancer. ${ }^{11}$ Fibrinogen levels greater than $600 \mathrm{mg} / \mathrm{dL}$ have also been associated with a diagnosis of malignant disease following acute ischemic stroke. ${ }^{11}$ Multiple ischemic lesions (acute or chronic) 
Box 2: Risks for occult cancer in acute ischemic stroke

\section{Clinical features}

- Older age $^{2}$

- Male sex ${ }^{2}$

- Lower body mass index

- History of smoking ${ }^{10}$

- History of heavy drinking ${ }^{10}$

- History of ischemic stroke (before current event) ${ }^{1}$

- History of cancer (past/nonactive) ${ }^{9}$

\section{Stroke features}

- Undetermined stroke etiology (after initial investigation) ${ }^{11}$

- Multiple vascular territories involved (on brain imaging) ${ }^{12}$

\section{Laboratory features}

- Low hemoglobin concentration ${ }^{8}$

- Low albumin level ${ }^{8}$

- Elevated D-dimer level ${ }^{1,8}$

- Elevated fibrinogen level ${ }^{1,11}$

- Elevated C-reactive protein level $\mathrm{l}^{1,8,11}$

- Elevated lactate dehydrogenase level ${ }^{12}$

involving more than 1 vascular territory on brain imaging are also suggestive of occult cancer..$^{12} \mathrm{~A}$ paroxysmal embolism may be suspected in people with a substantial patent foramen ovale on echocardiography, with or without associated deep vein thrombosis on lower extremity venous ultrasonography. Transesophageal echocardiography may be warranted to identify nonbacterial thrombotic endocarditis, as a transthoracic approach has limited sensitivity. ${ }^{3}$

The choice of antithrombotic therapy in cancer-associated stroke is individualized. Anticoagulation is indicated for people with atrial fibrillation, venous thromboembolism and nonbacterial thrombotic endocarditis. ${ }^{3,4}$ Treatment with low-molecularweight heparin or a direct oral anticoagulant such as edoxaban may reduce paraneoplastic hypercoagulability. ${ }^{4}$

Several features in our case led us to suspect occult cancer. Our patient was older, had a first-degree family history of cancer, had not been screened for colorectal cancer and had few known modifiable cerebrovascular risk factors. Her normocytic anemia and slightly elevated C-reactive protein level were compatible with low-grade systemic inflammation.

Our case shows the importance of considering occult cancer in ischemic stroke survivors. It highlights factors that may prompt additional investigations as part of a comprehensive approach to ischemic stroke.

\section{References}

1. Selvik HA, Thomassen L, Bjerkreim AT, et al. Cancer-associated stroke: the Bergen NORSTROKE study. Cerebrovasc Dis Extra 2015;5:107-13.

2. Qureshi Al, Malik AA, Saeed O, et al. Incident cancer in a cohort of 3247 cancer diagnosis free ischemic stroke patients. Cerebrovasc Dis 2015;39:262-8.

3. Dearborn JL, Urrutia VC, Zeiler SR. Stroke and cancer - a complicated relationship. J Neurol Transl Neurosci 2014;2:1039.

4. Navi BB, ladecola C. Ischemic stroke in cancer patients: a review of an underappreciated pathology. Ann Neurol 2018;83:873-83.

5. Wein T, Lindsay MP, Cote R, et al. Canadian stroke best practice recommendations: secondary prevention of stroke, sixth edition practice guidelines, update 2017. Int J Stroke 2018;13:420-43.

6. Kernan WN, Ovbiagele B, Black HR, et al. Guidelines for the prevention of stroke in patients with stroke and transient ischemic attack: a guideline for healthcare professionals from the American Heart Association/American Stroke Association. Stroke 2014;45:2160-236.

7. Published guidelines. Canadian Task Force on Preventive Health Care; updated 2019. Available: https://canadiantaskforce.ca/guidelines/published -guidelines/ (accessed 2020 Feb. 2).

8. Gon Y, Sakaguchi M, Takasugi J, et al. Plasma D-dimer levels and ischaemic lesions in multiple vascular regions can predict occult cancer in patients with cryptogenic stroke. Eur J Neurol 2017;24:503-8.

9. Quintas S, Rogado J, Gullon P, et al. Predictors of unknown cancer in patients with ischemic stroke. J Neurooncol 2018;137:551-7.

10. Aarnio K, Joensuu H, Haapaniemi $\mathrm{E}$, et al. Cancer in young adults with ischemic stroke. Stroke 2015;46:1601-6.

11. Cocho D, Gendre J, Boltes A, et al. Predictors of occult cancer in acute ischemic stroke patients. J Stroke Cerebrovasc Dis 2015;24:1324-8.

12. Kassubek R, Bullinger L, Kassubek J, et al. Identifying ischemic stroke associated with cancer: a multiple model derived from a case-control analysis. J Neurol 2017;264:781-91.

Competing interests: Mark Keezer has received speaker and advisory fees for Eisai Co., Elsevier, Sunovion, Novartis and UCB, and unrestricted educational grants and research grants from UCB and Eisai Co. Laura Gioia has received speaker fees and advisory board honoraria from Bayer, BMS-Pfizer and Servier, and investigator-initiated funding from Servier. No other competing interests were declared.

This article has been peer reviewed.

The authors have obtained patient consent.

Affiliations: Department of Neurosciences (Rioux, Keezer, Gioia), University of Montreal; Centre hospitalier de l'Université de Montréal (Rioux, Keezer, Gioia); Department of Social and Preventive Medicine (Keezer), University of Montreal, Montréal, Que.

Contributors: All of the authors contributed to the design and writing of the manuscript, approved the final version to be published and agreed to be accountable for all aspects of the work.

Correspondence to: Laura Gioia, laura.gioia@umontreal.ca 